\title{
Primary Central Nervous System Lymphoma: A Case of Solitary Brain Lesion
}

\author{
Halimat Lawal ${ }^{1}$, Nya Anwanane ${ }^{2}$, Anas Atrash ${ }^{1}$ \\ 1. Internal Medicine, University of Pittsburgh Medical Center Pinnacle, Harrisburg, USA 2. Internal Medicine, Oakland \\ University, Michigan, USA
}

Corresponding author: Halimat Lawal, lawalho@upmc.edu

\begin{abstract}
Primary central nervous system lymphoma (PCNSL) is an uncommon variant of extranodal non-Hodgkin's lymphoma (NHL) that involves the brain, leptomeninges, eyes, or spinal cord without evidence of systemic disease.
\end{abstract}

This article presents a case of a 63-year-old Caucasian female with high-grade B-cell lymphoma who was found to have a solitary brain lesion and no distant metastasis upon diagnosis. The patient had left-sided weakness and difficulty standing on presentation and was found to have a right frontal lobe mass with surrounding mild vasogenic edema and a small focal area of hemorrhage concerning for high-grade glioma versus lymphoma on MRI. However, pathology results revealed high-grade B-cell lymphoma.

The case reinforces the importance of working up any lesion suspicious for lymphoma/glioma. Diagnosis of high-grade B-cell lymphoma can be difficult based on morphological and cytological appearance due to varying gene expression and presentation at diagnosis. It can closely mimic diffuse B-cell lymphoma. Extensive workup including HIV serology, MRI imaging, evaluation for spinal cord involvement, and lumbar puncture (LP), to rule out cerebrospinal fluid (CSF) involvement, prior to initiating treatment needs to be done. The case also addresses high-grade methotrexate (MTX)-based chemotherapy as a treatment that improves mortality in patients with primary central nervous system (CNS) lymphoma.

Review began 05/10/2021 Review ended 06/20/2021 Published 06/27/2021

๑) Copyright 2021 Lawal et al. This is an open access article distributed under the terms of the Creative Commons Attribution License CC-BY 4.0., which permits unrestricted use, distribution, and reproduction in any medium, provided the original author and source are credited.
Categories: Internal Medicine, Oncology, Hematology

Keywords: primary cns lymphoma, solitary brain lesions, high grade lymphoma, lymphoma, cns lymphoma, brain tumors, b cell lymphoma, extra nodal non hodgkin lymphoma, lymphoma versus glioma

\section{Introduction}

Primary central nervous system lymphoma (PCNSL) is an uncommon variant of extranodal non-Hodgkin's lymphoma (NHL) that involves the brain, leptomeninges, eyes, or spinal cord without evidence of systemic disease. Here we present a case of a 63-year-old Caucasian female with high-grade B-cell lymphoma who was found to have a solitary brain lesion and no distant metastasis upon diagnosis.

\section{Case Presentation}

A 63-year-old Caucasian female with a past medical history (PMH) of arthritis presented originally to the hospital due to an abnormal MRI. The patient was having weakness on the left side for the past few weeks along with difficulty concentrating. Her symptoms started to worsen when she was leaning towards the left upon standing, veering to the left when driving, and had difficulty standing from a seated position. She was evaluated by her primary care physician (PCP) who ordered a brain MRI. The MRI showed a right frontal lobe mass with surrounding mild vasogenic edema and a small focal area of hemorrhage as seen in Figure 1. 


\section{Cureus}

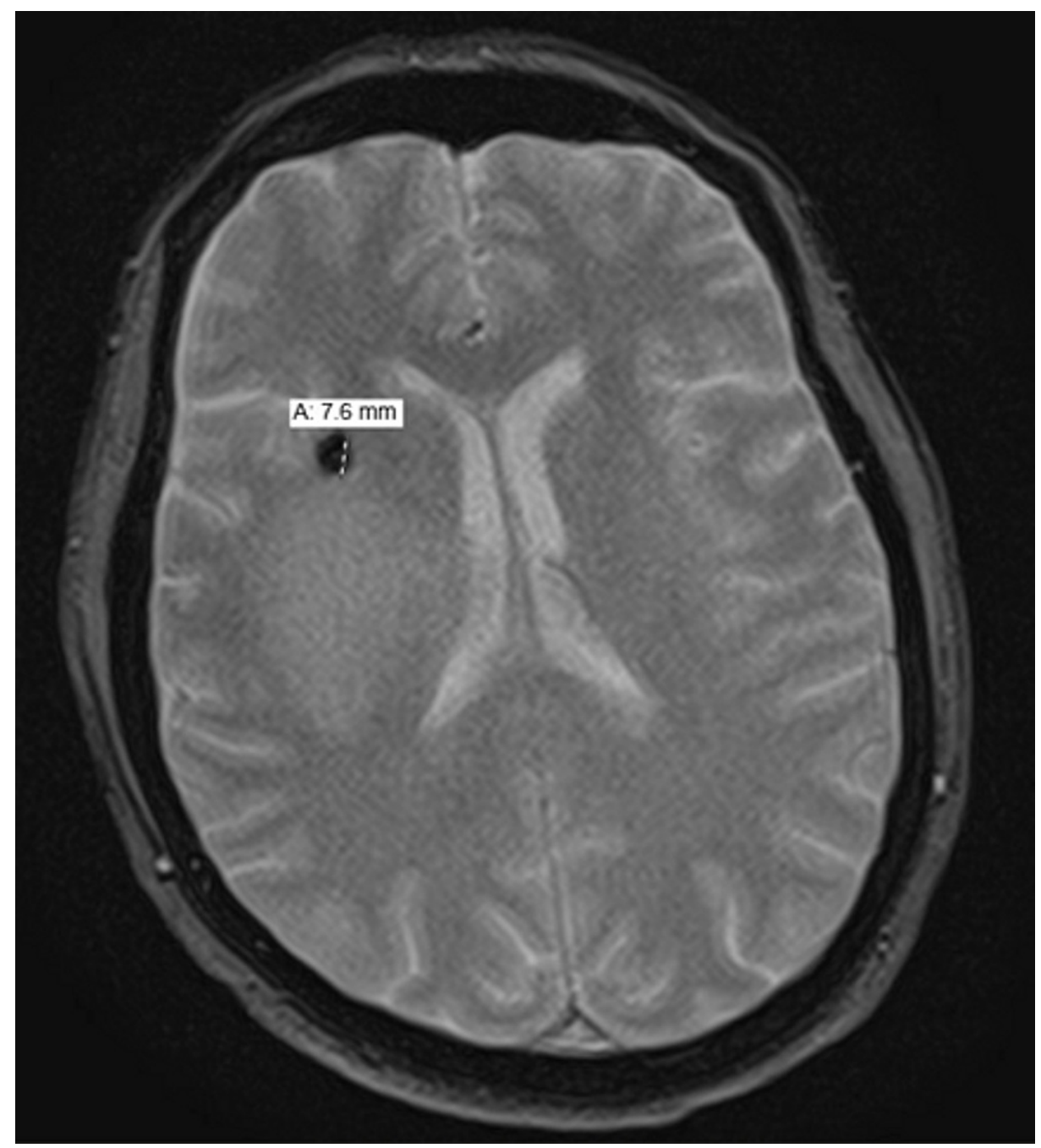

\section{FIGURE 1: Pre-op MRI showing mass with surrounding vasogenic}

edema.

These findings were concerning for high-grade glioma with other differentials including lymphoma and other causes. She was then sent to the hospital for further evaluation. Family history is significant for NHL in the father and brother diagnosed with NHL $\sim 15$ years ago, who are in remission. On initial exam, vitals were significant for mildly elevated blood pressure but otherwise unremarkable. On neurologic exam, the patient was notably alert and oriented $\mathrm{x}$, with intact speech but mild difficulty swallowing. She had mild left pronator drift, left-sided facial droop, left hemiparesis with $4 / 5$ strength on the left upper and lower extremity, and $5 / 5$ strength on the right upper and lower extremity. Sensations were intact in all extremities. She was started on $500 \mathrm{mg}$ of Keppra, every $12 \mathrm{~h}$ and $4 \mathrm{mg}$ of dexamethasone, every $8 \mathrm{~h}$. A CT chest/abdomen/pelvis was obtained which showed no evidence of metastatic disease. Neurosurgery evaluated the patient and she underwent right frontoparietal craniotomy and partial removal of the tumor. The patient had a post-surgical brain CT that showed small amounts of high attenuation material in gas bubbles along the track of the resection, likely representing residual acute blood (Figure 2). 


\section{Cureus}

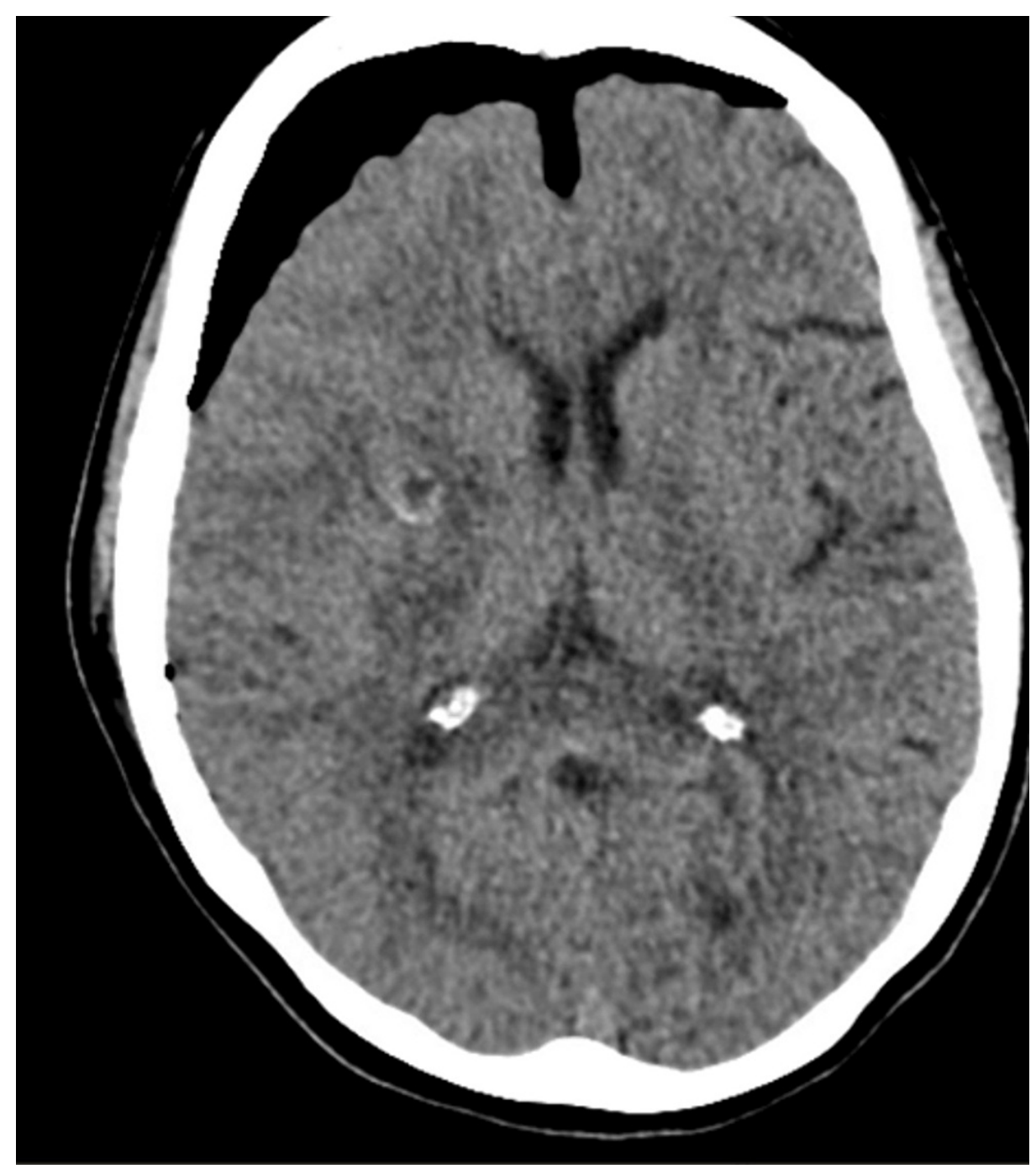

FIGURE 2: Post-op CT scan showing post-surgical changes and residual acute blood.

A follow-up MRI of the brain revealed that surgical changes of the right frontal lobe were present. There was a tract extending through the previously seen right frontal mass which had hemosiderin deposition and hemorrhagic changes surrounding the tract. It extended from the craniotomy site in the right frontal lobe to the right basal ganglia. The large right frontal lobe mass showed a slight decrease in size with the central portion debulked. However, there was still marked surrounding edema and residual enhancing brain parenchyma. In addition, previously seen abnormal edema, which extended across the midline, into the left frontal was still noted (Figure 3). 


\section{Cureus}

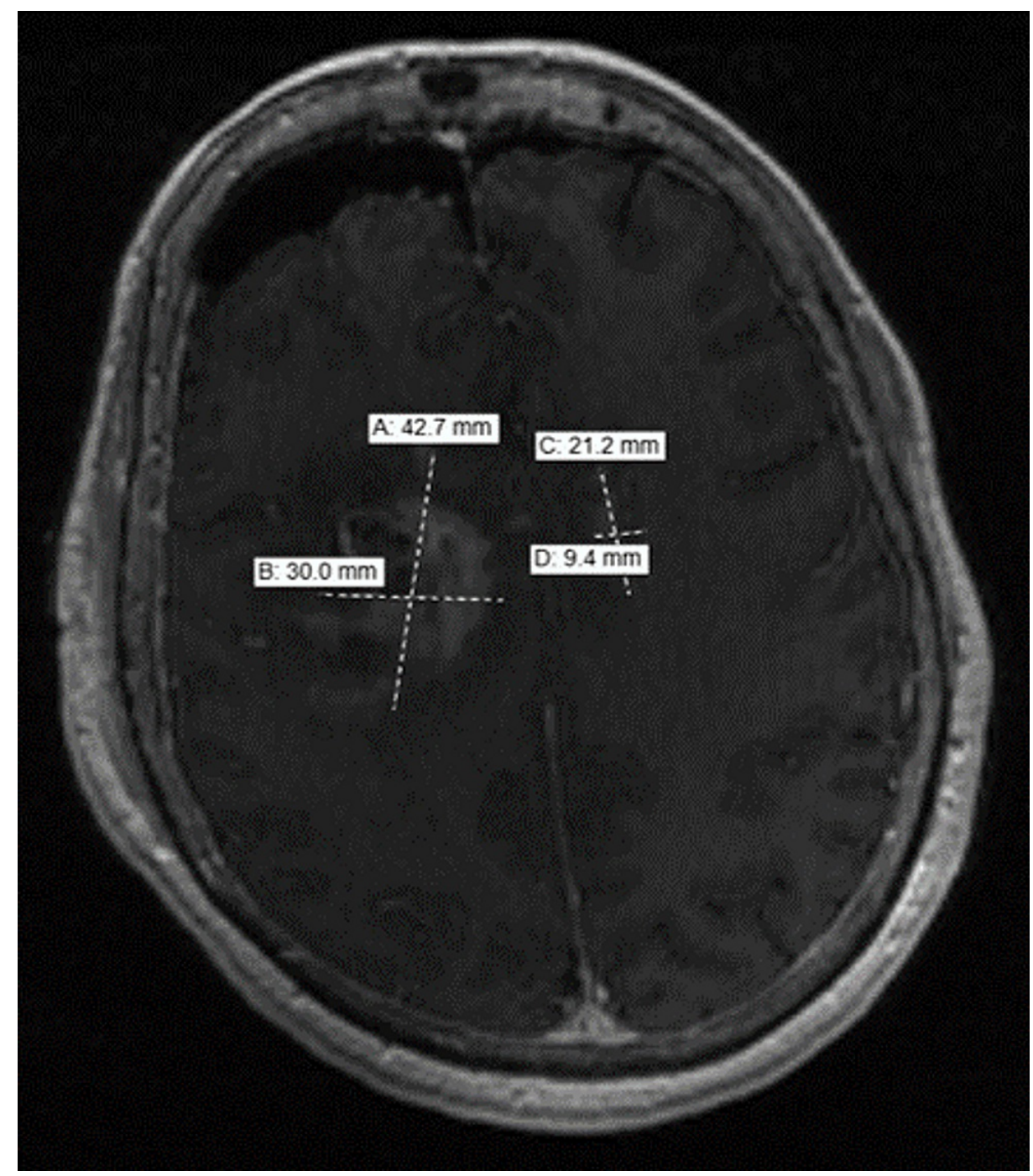

FIGURE 3: Post-op MRI showing debulked tumor with persistent surrounding edema.

Following IV contrast administration there was still prominent enhancement surrounding the surgical changes and the residual underlying mass in the right frontal lobe, which was infiltrative in appearance and still demonstrated enhancement. It measured about $3 \mathrm{~cm} \mathrm{x} 4.3 \mathrm{~cm}$ in diameter. The left-sided lesion also demonstrated enhancement, which measured about $2.1 \mathrm{~cm}$ x $9.4 \mathrm{~cm}$. Final tissue pathology revealed highgrade large B-cell lymphoma as seen in Figures 4-5. 


\section{Cureus}

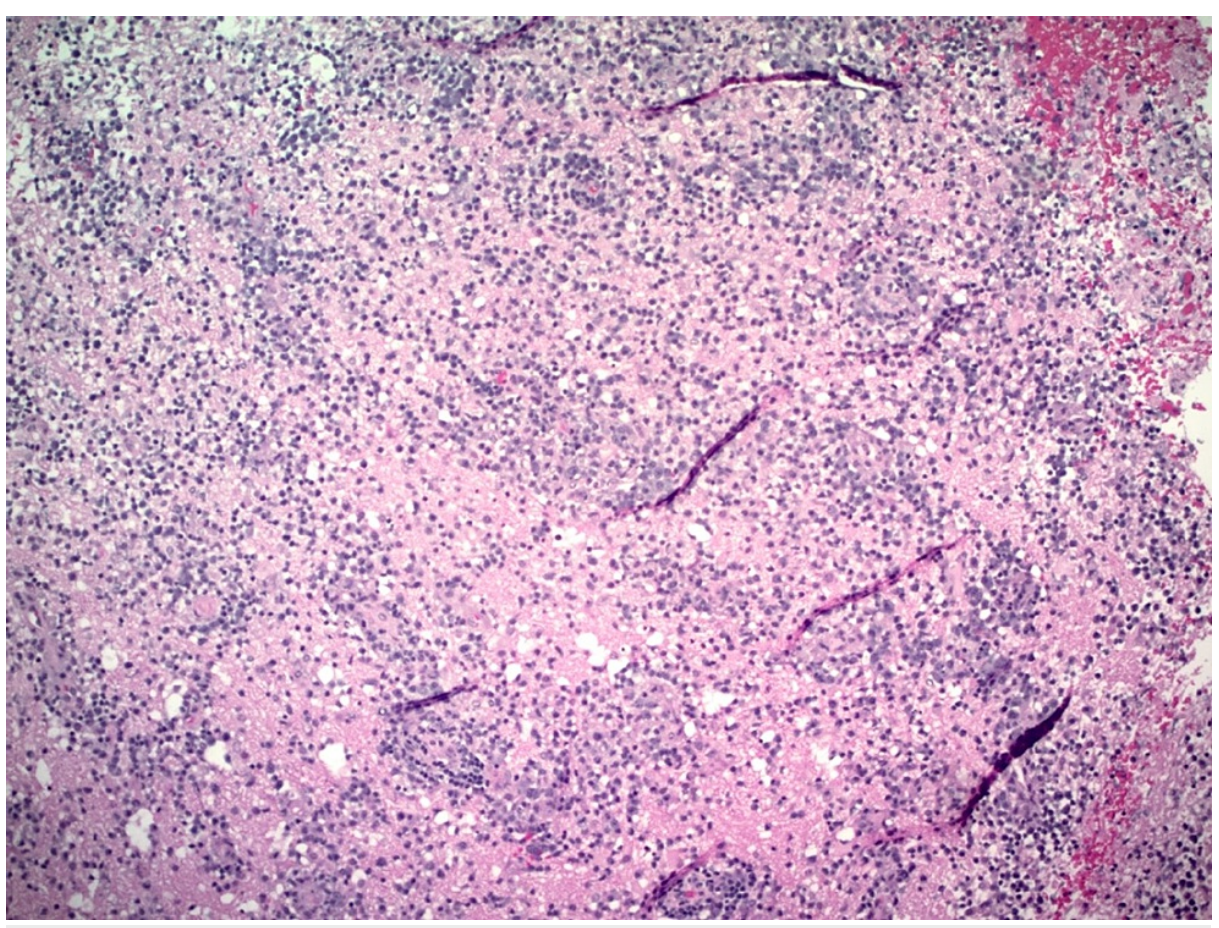

FIGURE 4: High-grade large B-cell lymphoma.

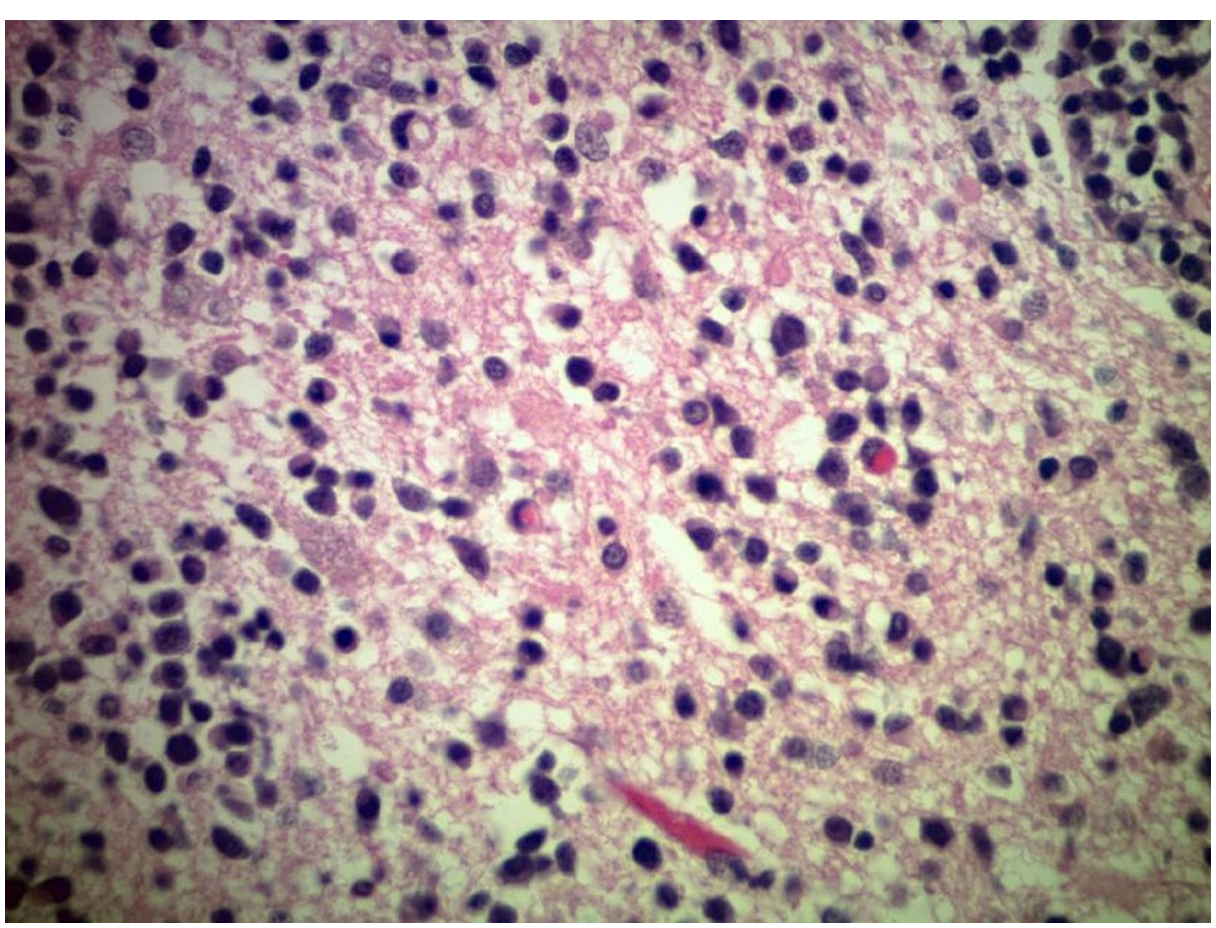

FIGURE 5: High-grade large B-cell lymphoma.

Immunoassay revealed atypical brain cells to be positive for CD20, PAX-5, BCL1, BCL6 (focal), MUM-1, with a high proliferation rate, estimated KI-67 >60\%. The cells are negative for: $\mathrm{CD} 3, \mathrm{CD} 5, \mathrm{CD} 10, \mathrm{CD} 15, \mathrm{CD} 30$, CD23, BCL1, CMYC (less than 30\%), CD34, TDT, and EBER. Flow cytometry analysis reported monoclonal Bcells with Lambda light chain restriction, consistent with B-cell lymphoproliferative disorder. The patient showed symptomatic improvement s/p resection and was discharged to a rehab facility. She continued on Keppra and dexamethasone with scheduled follow-up with Oncology for further management. On a followup visit, Oncology proposed therapy with high-dose methotrexate (MTX) with leucovorin rescue that would be administered every two weeks for 12 weeks, a total of six treatments. Prior to initiation of treatment, however, further studies were done to evaluate for metastasis including MRI and lumbar puncture (LP). LP 
was done to rule out cerebrospinal fluid (CSF) involvement prior to initiation of therapy and results were negative for malignant cells. MRI spine was also done which was negative for any lesions. The patient was scheduled to continue with a treatment plan as proposed and currently keeps improving symptomatically.

\section{Discussion}

This case-based pathology description will be classified as "high-grade B-cell lymphoma with BCL6 gene arrangement.” And based on the presentation of the patient considering no systemic involvement and overall history and presentation. This aligns with an extranodal NHL involving the brain primarily, which brings us to the topic of primary central nervous system (CNS) lymphoma.

Primary central nervous system lymphoma is an uncommon variant of extranodal NHL that involves the brain, leptomeninges, eyes, or spinal cord without evidence of systemic disease. As seen in this patient, the lesion is only involving the brain without the involvement of any other organ or systemic disease. The epidemiology and clinical presentation vary depending upon the immunocompetence of the patient. PCNSL represents approximately four percent of newly diagnosed primary CNS tumors, with an age-adjusted incidence rate of four cases per million persons per year [1-2]. The incidence in the general population rose from the 1960s to the 1990s, peaked in the mid-1990s, and then declined [1, 3-13]. Such changes were largely driven by PCNSL cases in men between the ages of 20 and 64 years [14]. Thus, the trend has been attributed in large part to changes in HIV incidence and management over the same time period. By contrast, the incidence rate in adults $>65$ years of age has steadily risen, even in the last decade [14]. Most cases of nonHIV-related PCNSL are diagnosed in patients between 45 and 65 years of age, with a median age at diagnosis in the fifth decade [3,15-17]. Sporadic PCNSL, in apparently immunocompetent individuals, has been reported in association with other diseases. Antecedent flu-like or gastrointestinal illnesses like gastroenteritis have been seen in up to $15 \%$ of patients and many patients with PCNSL [4]. In addition, patients with autoimmune diseases such as rheumatoid arthritis, systemic lupus erythematosus, Sjögren syndrome, myasthenia gravis, sarcoidosis, and vasculitis may be predisposed to develop either disease- or therapy-related PCNSL [18]. Several distinctive radiographic features suggest the diagnosis of PCNSL in immunocompetent individuals. Approximately 50\%-70\% of immunocompetent patients with PCNSL develop solitary lesions [19], with the remainder (approximately 25\%) developing the multifocal disease.

Periventricular lesions (e.g., thalamus, basal ganglia, and corpus callosum) are most common (60\%) followed by lesions in the frontal, parietal, temporal, and occipital lobes in $20 \%, 18 \%, 15 \%$, and $4 \%$ of patients, respectively [20]. The radiographic lesion tends to be a solitary nonhemorrhagic mass, situated in the deep white matter adjacent to the ventricular surface. The borders are sharply circumscribed in the majority of lesions (87\%), but maybe ill defined (15\%) [20]. Although mild surrounding edema is present in the majority of cases, it is usually less profound than that which accompanies metastatic foci of carcinoma. Mass effect and tumor edema are seen in over half of the cases. Primary CNS Hodgkin's lymphoma and low-grade PCNSL are extremely rare. The latter has a better long-term outcome than aggressive histology PCNSL. Solitary lesions of the brain concerning lymphoma can have varying presentations and management will require a thorough evaluation of risk factors.

The most common histopathologic subtype of PCNSL is diffuse large B-cell lymphoma (DLBCL). As an example, a series of 33 patients with PCNSL reported that all of these tumors were DLBCL, with the vast majority being of the centroblastic subtype [17]. The majority was positive for BCL6, MUM1, and BCL2; all were negative for CD138. Similar results were seen in a series of 51 patients with non-HIV-related PCNSL and a histologic diagnosis of DLBCL. Positivity for MUM1, BCL6, BCL2, CD10, or CD138 was seen in 84\%, $61 \%, 49 \%, 18 \%$, and $0 \%$, respectively. High-dose MTX-based chemotherapy is a standard component of initial therapy for PCNSL. In aggregate, the available data suggest that chemotherapy regimens that include high-dose systemic MTX are more effective against PCNSL than either radiation alone or regimens that do not contain MTX. The addition of rituximab to MTX-based regimens may provide additional benefit and is well tolerated.

\section{Conclusions}

Primary CNS lymphoma is a type of high-grade B-cell lymphoma and is a rare disease. Diagnosis can be difficult based on morphological and cytological appearance due to varying gene expression and presentation at diagnosis. It can closely mimic DLBCL which is why it was originally classified as such but it can also present as a solitary extranodal lesion as seen in primary CNS lymphoma which we see in this case. It is, therefore, essential to work up any lesion suspicious for lymphoma/glioma extensively. Multiple lab work including HIV serology, imaging with MRI to evaluate for spinal cord involvement, and LP to rule out CSF involvement needs to be done prior to initiating the treatment. Slit-lamp and ophthalmology evaluation also needs to be done to rule out eye involvement. Therapy will highly depend on tissue pathology and the extent of involvement at diagnosis. Rituximab with high-dose MTX-based chemotherapy has been proven to improve prognosis in PCNSL.

\section{Additional Information}

\section{Disclosures}

Human subjects: Consent was obtained or waived by all participants in this study. Conflicts of interest: In 
compliance with the ICMJE uniform disclosure form, all authors declare the following: Payment/services info: All authors have declared that no financial support was received from any organization for the submitted work. Financial relationships: All authors have declared that they have no financial relationships at present or within the previous three years with any organizations that might have an interest in the submitted work. Other relationships: All authors have declared that there are no other relationships or activities that could appear to have influenced the submitted work.

\section{References}

1. Hoffman S, Propp JM, McCarthy BJ: Temporal trends in incidence of primary brain tumors in the United States, 1985-1999. Neuro Oncol. 2006, 8:27.

2. Villano JL, Koshy M, Shaikh H, et al.: Age, gender, and racial differences in incidence and survival in primary CNS lymphoma. Br J Cancer. 2011, 105:1414.

3. Miller DC, Hochberg FH, Harris NL, et al.: Pathology with clinical correlations of primary central nervous system non-Hodgkin's lymphoma. The Massachusetts General Hospital experience 1958-1989. Cancer. 1994, $74: 1383$.

4. Hochberg FH, Miller DC: Primary central nervous system lymphoma. J Neurosurg. 1988, 68:835.

5. Corn BW, Marcus SM, Topham A, et al.: Will primary central nervous system lymphoma be the most frequent brain tumor diagnosed in the year 2000?. Cancer. 1997, 79:2409.

6. Ahsan H, Neugut AI, Bruce JN: Trends in incidence of primary malignant brain tumors in USA, 1981-1990 . Int J Epidemiol. 1995, 24:1078.

7. Schabet M: Epidemiology of primary CNS lymphoma. J Neurooncol. 1999, 43:199.

8. Haldorsen IS, Krossnes BK, Aarseth JH, et al.: Increasing incidence and continued dismal outcome of primary central nervous system lymphoma in Norway 1989-2003: time trends in a 15-year national survey. Cancer. 2007, 110:1803.

9. Olson JE, Janney CA, Rao RD, et al.: The continuing increase in the incidence of primary central nervous system non-Hodgkin lymphoma: a surveillance, epidemiology, and end results analysis. Cancer. 2002, 95:1504.

10. Krogh-Jensen M, d'Amore F, Jensen MK, et al.: Incidence, clinicopathological features and outcome of primary central nervous system lymphomas. Population-based data from a Danish lymphoma registry. Danish Lymphoma Study Group, LYFO. Ann Oncol. 1994, 5:349.

11. Krogh-Jensen M, D'Amore F, Jensen MK, et al.: Clinicopathological features, survival and prognostic factors of primary central nervous system lymphomas: trends in incidence of primary central nervous system lymphomas and primary malignant brain tumors in a well-defined geographical area. Population-based data from the Danish Lymphoma Registry, LYFO, and the Danish Cancer Registry. Leuk Lymphoma. 1995, 19:223.

12. Aalen OO, Farewell VT, De Angelis D, et al.: New therapy explains the fall in AIDS incidence with a substantial rise in number of persons on treatment expected. AIDS. 1999, 13:103.

13. Kadan-Lottick NS, Skluzacek MC, Gurney JG: Decreasing incidence rates of primary central nervous system lymphoma. Cancer. 2002, 95:193.

14. O'Neill BP, Decker PA, Tieu C, Cerhan JR: The changing incidence of primary central nervous system lymphoma is driven primarily by the changing incidence in young and middle-aged men and differs from time trends in systemic diffuse large B-cell non-Hodgkin's lymphoma. Am J Hematol. 2013, 88:997.

15. Fine HA, Mayer RJ: Primary central nervous system lymphoma. Ann Intern Med. 1993, 119:1093.

16. Herrlinger U, Schabet M, Clemens M, et al.: Clinical presentation and therapeutic outcome in 26 patients with primary CNS lymphoma. Acta Neurol Scand. 1998, 97:257.

17. Braaten KM, Betensky RA, de Leval L, et al.: BCL-6 expression predicts improved survival in patients with primary central nervous system lymphoma. Clin Cancer Res. 2003, 9:1063.

18. Bhagavathi S, Wilson JD: Primary central nervous system lymphoma. Arch Pathol Lab Med. 2008, 132:1830.

19. Rock JP, Cher L, Hochberg FH, et al.: Primary CNS lymphoma. Neurological Surgery, 4th ed. Yomans JR (ed): WB Saunders, Philadelphia; 1966. 2688.

20. Bataille B, Delwail V, Menet E, et al.: Primary intracerebral malignant lymphoma: report of 248 cases . J Neurosurg. 2000, 92:261. 